\title{
Psychology Students’ Beliefs about Integrating Complementary and Alternative Therapy (CAT) into Their Future Psychology Practice
}

\author{
Lee-Ann M. Wilson ${ }^{1}$, Kyra Hamilton ${ }^{2}$, Katherine M. White ${ }^{2}$ \\ ${ }^{1}$ School of Public Health, Queensland University of Technology, Brisbane, Australia \\ ${ }^{2}$ School of Psychology and Counselling, Queensland University of Technology, Brisbane, Australia \\ Email: lm.wilson@qut.edu.au
}

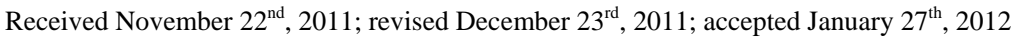

\begin{abstract}
We investigated whether belief-based differences exist between students who have strong and weak intentions to integrate complementary and alternative therapy (CAT) into future psychology practice by recommending CAT or specific CAT practitioners to clients. A cross-sectional methodology was used. Psychology undergraduate students $(N=106)$ participated in a paper-based questionnaire design to explore their underlying beliefs related to CAT integration. The study was undertaken at a major university in Queensland, Australia. The theory of planned behaviour belief-based framework guided the study. Multivariate analyses of variance examined the influence of behavioural, normative, and control beliefs on the strong and weak intention groups. A multiple regression analysis investigated the relative importance of these belief sets for predicting intentions. We found that clear differences emerged between strong and weak intenders on behavioural and normative beliefs, but not control beliefs. Strong intenders perceived the positive outcomes of integrating CAT, such as being able to offer clients a more holistic practice and having confidence in the practitioners/practices, as more likely to occur than weak intenders, and perceived the negative outcome of compromising my professional practice as less likely. Strong intenders were more likely than weak intenders to perceive that a range of important referents (e.g., clients) would think they should integrate CAT. Results of the regression analysis revealed the same pattern of results in that behavioural and normative beliefs, but not control beliefs, significantly predicted intentions. The findings from this study can be used to inform policy and educational initiatives that aim to encourage CAT use in psychology practice.
\end{abstract}

Keywords: Integrating Complementary Therapy; Psychology Practice; Psychology Student; Theory of Planned Behaviour; Beliefs

\section{Introduction}

Recent research suggests that many individuals with mental health disorders seek the advice of naturopaths, or combine yoga, meditation, or herbal therapies with their traditional psychotherapy (Page, Jones, \& Wilson, 2004; Australian Bureau of Statistics, 2004-2005). While significant research has been conducted exploring medical practitioners' attitudes and perceptions toward complementary and alternative therapies (CAT) (Halcon, Chlan, Kreitzer, \& Leonard, 2003; Hyland, Lewith, \& Westoby, 2003; Lewith, Hyland, \& Shaw, 2002), there is an absence of similar investigations within the field of psychology. Although some previous research has attempted to explore differences between medical and social science students' (predominately psychology students) attitudes toward complementary medical practitioners and medical treatment preferences (Yardley, Fahmy, Jamie, \& Furnham, 1999), no previous study has tried to demonstrate the key perceptions underlying psychology students' decisions regarding integrating CAT into their future professional practice.

Current research suggests that psychologists are integrating CAT into psychological practice via recommending CAT to clients, offering clients referrals to CAT practitioners, or ac- cessing training in CAT therapies to integrate these therapies into practice (Bassman \& Uellendahl, 2003). Previous research, although somewhat limited, has found that psychologists are recommending CAT generally at relatively high rates and at significantly higher rates than they are offering specific referrals to CAT practitioners (Bassman \& Uellendahl, 2003). However, little is known about how psychology students, who are the next cohort of health professionals and who will be in a position to direct future initiatives in the field, perceive integrating CAT into psychology practice. A qualitative pilot study examining psychology students' and practicing psychologists' attitudes and beliefs about CAT integration revealed that students are generally positive about CAT integration but indicated that they were largely unaware of the professional implications of the various integration choices, and their responses did not vary much between the different integration scenarios (i.e., they saw much the same implications between training themselves in CAT, recommending CAT to clients, and referring clients to CAT practitioners) (Wilson \& White, 2011). Students focused on the potential benefits to clients, such as being able to offer a broader range of treatment options. Given that previous research has found examining attitudes and beliefs useful in understanding CAT practices in general (Chua \& Furnham, 2008), having an understanding of the ways in which 
underlying beliefs guide psychology students' decisions about whether to integrate CAT into future practice might also be useful to inform both clinical practice policy and educational initiatives.

The Theory of Planned Behaviour (TPB; Ajzen, 1991) is a well validated decision-making model that allows for an examination of people's underlying beliefs and has much support in the social and health domains (see Armitage \& Christian, 2003). According to the TPB, intention to perform a given behaviour and the individual's actual control over performing the behaviour predict behavioural performance. Intention, in turn, is influenced by the degree to which a person is in favour of performing a particular behaviour (attitude), the degree to which a person feels social pressure to perform a behaviour (subjective norms), and the degree to which a person feels they have control over performing the behaviour (perceived behavioural control) (Ajzen, 1991). Underlying the TPB is the assumption that the antecedents of attitude, subjective norms, and perceived behavioural control are corresponding behavioural (costs and benefits), normative (pressure to comply with important others' approval/disapproval), and control beliefs (motivating or inhibiting factors), respectively, that reflect an individual's intention and subsequent behaviour (Ajzen, 1991). Assessing the belief-based determinants of attitude, subjective norms, and perceived behavioural control allows researchers to establish the beliefs that differentiate those who perform a given behaviour from those who do not. A number of studies have explored these underlying beliefs in order to increase understanding of health behaviours, including CAT practices (O’Conner \& White, 2009; Pawlak et al., 2008; Wilson \& White, 2008).

Using the TPB as a theoretical framework, this study aims to investigate the beliefs that differentiate those psychology students who have strong and weak intentions to integrate CAT into future psychology practice by either recommending CAT to clients or referring clients to specific CAT practitioners. Specifically, the study assessed psychology students' beliefs relating to the costs and benefits associated with recommending or referring CAT to clients (behavioural beliefs), beliefs about which important referents would approve or disapprove of their recommending or referring CAT (normative beliefs), and beliefs about inhibiting factors (control beliefs) for recommending or referring CAT to clients.

\section{Method}

\section{Participants}

Participants $(N=106)$ were psychology undergraduate students from a major Australian university. The majority of the students was enrolled in first year psychology units (89\%) and indicated a preference of clinical (42\%) or counseling (32\%) psychology as their future area of professional interest. The study comprised of 77 females and 29 males and the mean age of participants was 24.55 years $(S D=10.46$ years $)$.

\section{Measures}

The target behaviour assessed students' beliefs about integrating CAT into future psychology practice by either recommending CAT to clients or referring clients to specific CAT practitioners. The two behaviours, recommending and referring, were combined as pilot research utilising semi- structured interviews indicated that students did not differentiate between the professional implications or clients outcomes associated with these two practices (Wilson \& White, 2011). CAT was operationalised as: “A group of diverse medical and health care systems, practices, and products that are not presently considered to be part of conventional medicine”. This definition is consistent with the National Centre for Complementary and Alternative Medicines (2006). The pilot study which involved both practicing psychologists and psychology students was conducted $(N=12$; 6 psychologists and 6 students) to elicit the salient behavioural, normative, and control beliefs (8) as recommended by Fishbein and Ajzen (1975). Using content analysis, the most commonly reported advantages (e.g., being able to offer clients a more holistic practice) and disadvantages (e.g., not being able to follow up client progress with CAT), referents approving or disapproving (e.g., employers), and factors preventing integrating CAT into future psychology practice by recommending or referring CAT (e.g., lack of knowledge about relevant CATs) reported by students were used to assess the behavioural, normative, and control belief-based measures, respectively, in the present study.

All belief-based items were scored on 7-point Likert scales, scored extremely unlikely (1) to extremely likely (7). To assess behavioural beliefs, participants were asked to rate how likely two costs and two benefits would result if they integrated CAT into a future psychology practice by recommending CAT or specific CAT practitioners to clients. For normative beliefs, participants were asked to rate how likely four referents would think they should integrate CAT into a future psychology practice by recommending CAT or specific CAT practitioners to clients. Participants indicating how likely internal and external factors would prevent them from integrating CAT into a future psychology practice by recommending CAT or specific CAT practitioners to clients assessed control beliefs. It should be noted that internal consistency is not a necessary feature of belief composites as no assumption is made that the salient beliefs are internally consistent (Ajzen, 1991); thus, alpha coefficients were not computed for the behavioural, normative, and control belief-based measures. For a full listing of the belief-based items, see Table 1. The outcome measure of intention was measured on a single item scale. Students were asked to respond to the question: "I intend (1), do not intend (7) to integrate CAT into a future psychology practice by recommending CAT or specific CAT practitioners to clients?” For analyses, intention scores were recoded such that higher scores were associated with stronger intentions. Furthermore, for belief-based analyses, responses were recoded to indicate those who had weak (scores 1.0 to 4.0 ) and strong intentions (scores 4.0 to 7.0 ) to perform the target behaviour.

\section{Procedure}

Prior to any data collection, the university human research ethics committee approved the study. Students were informed about the opportunity to participate in the study during one of their regularly scheduled classes. At the end of the class, students who were willing to participate were able to complete and return their survey before leaving. The student participants were offered a small number of credit points towards a research participation mark in their psychology class as a thank you for 
their involvement.

\section{Results}

\section{Belief-Based Analyses}

Behavioural, normative, and control beliefs were examined to identify those beliefs differentiating between students who had strong and weak intentions to integrate CAT into a future psychology practice by recommending CAT or specific CAT practitioners to clients. Three one-way multivariate analyses of variance (MANOVAs) were conducted with intentions to perform the target behaviour as the independent variable and the belief-based items as the dependent variables. Overall, Wilks' Lambdas were significant for behavioural $(F(4,101)=7.59, p$ $\left.<.001, \eta^{2}=.23\right)$ and normative beliefs $(F(4,101)=4.80, p$ $\left.=.001, \eta^{2}=.16\right)$, but not for control beliefs $(F(4,101)=.28, p$ $=.891)$. To explore further where differences existed between the groups for behavioural beliefs and normative beliefs, dependent variables were examined at the univariate level. Bonferonni adjustments were used to control for familywise type 1 error (refer to Table 1). For behavioural beliefs, univariate analyses revealed that strong intenders perceived the positive outcomes of integrating CAT, such as be able to offer clients a more holistic practice and having confidence in the practitioners/prac-

Table 1.

Mean differences in behavioural, normative, and control beliefs.

\begin{tabular}{|c|c|c|}
\hline Intentions & $\begin{array}{c}\text { Weak } \\
\text { Intenders }\end{array}$ & $\begin{array}{l}\text { Strong } \\
\text { Intenders }\end{array}$ \\
\hline Behavioural beliefs & $N=58$ & $N=48$ \\
\hline Being able to offer a more holistic practice & 4.67 & $5.73^{* * *}$ \\
\hline $\begin{array}{l}\text { Not being able to follow up client progress } \\
\text { with CAT }\end{array}$ & 3.97 & 3.56 \\
\hline $\begin{array}{l}\text { Having confidence in the practitioners/ } \\
\text { practices that are being recommended }\end{array}$ & 4.40 & $5.44^{* * *}$ \\
\hline Compromising my professional reputation & 3.86 & $3.08^{* * *}$ \\
\hline \multicolumn{3}{|l|}{${ }^{* * *} p<.012$} \\
\hline Normative beliefs & $n=57$ & $n=48$ \\
\hline Your clients & 4.40 & $5.19^{* * *}$ \\
\hline Complementary or alternative practitioners & 5.44 & 5.77 \\
\hline Your employers & 3.98 & $4.83^{* * *}$ \\
\hline Professional organisations & 3.84 & $4.56^{* * *}$ \\
\hline \multicolumn{3}{|l|}{${ }^{* * *} p<.012$} \\
\hline Control beliefs & $n=58$ & $n=48$ \\
\hline $\begin{array}{l}\text { Lack of knowledge about appropriate } \\
\text { practitioners }\end{array}$ & 5.31 & 5.04 \\
\hline Lack of knowledge about relevant CATs & 5.34 & 5.17 \\
\hline $\begin{array}{l}\text { Lack of scientific evidence in support } \\
\text { of CAT }\end{array}$ & 4.91 & 4.71 \\
\hline $\begin{array}{l}\text { Absence of clear legal and professional } \\
\text { bodies guidelines }\end{array}$ & 5.00 & 4.83 \\
\hline${ }^{* * *} p<.012$ & & \\
\hline
\end{tabular}

tices, as more likely to occur than weak intenders, and were less likely to perceive that integrating CAT would compromise their professional practice. For normative beliefs, univariate analyses indicated that strong intenders were more likely than weak intenders to report the important referents of "your clients", "your employers", and "professional organisations" would think they should integrate CAT into a future psychology practice by recommending CAT or specific CAT practitioners to clients.

\section{Analysis Predicting Intentions}

The MANOVA analyses identified how the strong and weak intenders differed in terms of their beliefs. However, these analyses did not allow an assessment of which beliefs are most influential for students' intentions to integrate CAT into a future psychology practice by recommending CAT or specific CAT practitioners to clients. A multiple regression analysis, therefore, was conducted to assess the relative importance of the behavioural, normative, and control beliefs for predicting intentions. The dependent measure was based on a continuous scale reflecting intentions (as described in the Method Section). Together, the belief-based measures significantly predicted intentions, $F(3,101)=17.62, p<.001$, accounting for $34 \%$ of the variance of intentions to integrate CAT into a future psychology practice by recommending CAT or specific CAT practitioners to clients (refer to Table 2). Behavioural and normative beliefs, but not control beliefs, were significant predictors of intentions. Thus, the more likely students perceived benefits associated with recommending or referring CAT and the more important referents approved of recommending or referring, the more students were to intend to integrate CAT into a future psychology practice by recommending CAT or specific CAT practitioners to clients.

\section{Discussion}

The present study aimed to investigate whether belief-based differences exist between those students who have strong and weak intentions to integrate CAT into a future psychology practice by recommending CAT or specific CAT practitioners to clients. We found that the behavioural and normative beliefs, but not control beliefs, differed significantly between the intention groups. Additionally, the analysis examining the relative importance of these belief sets in predicting intentions showed the same pattern of results.

The findings of this study help us to understand the relationship between beliefs and students' intentions to integrate CAT into future psychology practice. This understanding, in turn, has important practical implications for those involved in clinical

Table 2.

Regression analysis predicting intentions.

\begin{tabular}{lccccc}
\hline Variable & $R$ & $R^{2}$ & $F$ & $d f$ & $\beta$ \\
\hline Intentions & .59 & .34 & 17.62 & 3,101 & \\
Behavioural beliefs & & & & & $.42^{* * *}$ \\
Normative beliefs & & & & & $.23^{* *}$ \\
Control beliefs & & & & & -.14 \\
\hline
\end{tabular}

${ }^{* *} p=.017,{ }^{* * *} p<.001$. 
practice policy-making and educational initiatives aimed at influencing CAT use within psychology practice. First, the finding that behavioural beliefs differed significantly between the groups suggests that policy and educational initiatives aimed towards promoting CAT integration into psychology practice should focus on increasing students' positive attitudes. Specifically, these should highlight that recommending or referring CAT would result in the ability to offer clients a more holistic practice, or building their confidence in the practitioners/practices that are being recommended, and reassuring them that compromising their professional reputation is not necessarily an outcome. Second, the differences in normative beliefs suggest that strong intenders were more likely than weak intenders to report those important referents (namely, clients, employers, and professional organizations) would think they should integrate CAT into future psychology practice by recommending CAT or specific CAT practitioners to clients. Consequently, policy and educational initiatives should focus on strategies that explicitly show clients, employers, and professional organizations approving of integrating CAT into psychology practice. Finally, inspection of the control beliefs revealed that strong and weak intenders were equally likely to perceive certain preventive factors (e.g., lack of knowledge about appropriate practitioners) as inhibiting their control over future integration. Accordingly, strategies that focus on discouraging aspects of integrating CAT into psychological practice (e.g., compromising professional reputation) may not be useful to help students overcome barriers that might prevent them from intending to integrate these behaviours into their future practice.

The results of the current study are broadly consistent with previous TPB belief-based studies investigating psychologists' intentions to integrate CAT (see Wilson \& White, 2008), where a range of behavioural, normative, and control beliefs influenced CAT decision-making. Practicing psychologists, however, have established professional training and experience and will be strongly influenced by past behaviours and norms; thus, psychologists' beliefs about CAT cannot be used to fully explain the beliefs of psychology students. This argument is supported by the finding that, in contrast with literature examining psychologists' beliefs (Wilson \& White, 2008), we did not find support for differences between strong and weak intenders for control beliefs. This discrepancy in findings may be attributable to the professional practice experience of the psychologists as students may not yet fully understand the potential barriers of integrating CAT into clinical practice. For example, psychology students may not have been exposed to professional codes of conduct that might potentially constrain the use of CAT in psychology practice.

The results of the regression analysis support the MANOVA findings in that behavioural and normative beliefs, but not control beliefs, predict students' intentions to integrate CAT into a future psychology practice by recommending CAT or specific CAT practitioners to clients, accounting for a reasonable amount of variance (34\%). These findings suggest that the more students perceive that there are benefits associated with recommending or referring CAT and the more they think that important referents approve of their recommending or referring CAT, the more students will intend to integrate CAT into a future psychology practice by recommending CAT or specific CAT practitioners to clients. In light of these finding, policy and educational initiatives aimed at promoting students' behavioural intentions toward CAT use in psychology practice should place emphasis on highlighting the behavioural and normative beliefs, and not necessarily on targeting the underlying control beliefs.

Some caution should be adopted in interpreting the results of this study. First, the sample size was small and may have been subject to self selection bias. Second, there was an uneven representation of female and male students. Although this imbalance reflects the higher proportion of females studying undergraduate psychology in Australia, it is important to keep in mind that our findings are primarily reflecting the view of female students. It is possible that male students may have different beliefs about CAT integration, at least as can be inferred from reported levels of personal use (MacLennan, Myers, \& Taylor, 2006; Satia-Abouta et al., 2003). Future research should incorporate a larger sample size in order to investigate this possibility. The broad definition of CAT used in the current study may have limited the responses toward recommending and referring CAT. The underlying beliefs for subcategories of CAT might show some differences to the beliefs identified in this study. For example, students' underlying beliefs regarding alternative therapies that have greater scientific evidence to support their usage (e.g., St. John's Wort, Folate, vitamin B12, and amino acids such as phenalalanine and S-Adenosylmethonine (SAMe); Jorm, Christensen, Griffiths, \& Rodgers, 2002) might be different for beliefs held toward alternative therapies that are less supported in the literature. Finally, we did not include a measure of the perceptions of public readiness and it might be interesting for future research to investigate a possible relationship between students' perceived public readiness to accept psychologists' integration of CAT and both students' own belief in CAT and their intention to use CAT in future practice.

Overall, by adopting a TPB belief-based approach, this study provides important practical information that can be used to inform policy and educational initiatives which aim to influence the integration of CAT in psychology practice. Specifically, the findings suggest that attention to advantages and disadvantages of integrating CAT, such as being able to offer clients a more holistic practice and compromising professional practice, as well as considering the social approval of clients, employers, and professional organisations in understanding CAT decision-making, may assist in improving students' intentions to integrate CAT into future psychology practice by recommending CAT or specific CAT practitioners to clients. Given the rise of CAT use for mental health disorders, this preliminary research examining CAT use in psychology practice should inform future research efforts to continue to investigate the motives and decision-making processes for integrating CAT within the field of psychology. This further research could include assessing the CAT integration beliefs and actions of practising psychologists and incorporating longitudinal designs to determine how students' attitudes and beliefs might change over time, especially in response to increased knowledge and professional experience.

\section{REFERENCES}

Ajzen, I. (1991). The theory of planned behavior. Organizational Behavior and Human Decision Processes, 50, 179-211. doi:10.1016/0749-5978(91)90020-T

Armitage, C. J., \& Christian, J. (2003). From attitudes to behaviour: Basic and applied research on the theory of planned behaviour. Cur- 
rent Psychology, 22, 187-195.

doi:10.1007/s12144-003-1015-5

Australian Bureau of Statistics (2004-2005). 4364.0 National Health Survey: Summary. URL (last checked 1 October 2006).

http://www.abs.gov.au/AUSSTATS/abs@.nsf/DetailsPage/4364.020 04-05?OpenDocument

Bassman, L. E., \& Uellendahl, G. (2003). Complementary/alternative medicine: Ethical, professional, and practical challenges for psychologists. Professional Psychology: Research and Practice, 34, 264-270. doi:10.1037/0735-7028.34.3.264

Chua, S. A., \& Furnham, A. (2008). Attitudes and beliefs towards complementary and alternative medicine (CAM): A cross-cultural approach comparing Singapore and the United Kingdom. Complementary Therapies in Medicine, 16, 247-253.

doi:10.1016/j.ctim.2008.02.009

Fishbein, M., \& Ajzen, I. (1975). Belief, attitude, intention, and behavior: An introduction to theory and research. Reading, MA: Addison-Wesley.

Halcon, L. L., Chlan, L. L., Kreitzer, M. J., \& Leonard, B. J. (2003). Complementary therapies and healing practices: Faculty/student beliefs and attitudes and the implications for nursing education. Journal of Professional Nursing, 19, 387-397. doi:10.1016/S8755-7223(03)00133-9

Hyland, M. E., Lewith, G. T., \& Westoby, C. (2003). Developing a measure of attitudes: The holistic complementary and alternative medicine questionnaire. Complementary Therapies in Medicine, 11, 33-38. doi:10.1016/S0965-2299(02)00113-9

Jorm, A. F., Christensen, H., Griffiths, K. M., \& Rodgers, B. (2002). Effectiveness of complementary and self-help treatments for depression. Medical Journal of Australia, 176, S84-S95.

Lewith, G. T., Hyland, M. E., \& Shaw, S. (2002). Do attitudes toward and beliefs about complementary medicine affect treatment outcomes? American Journal of Public Health, 92, 1064-1067. doi:10.2105/AJPH.92.10.1604

MacLennan, A. H., Myers, S. P., \& Taylor, A. W. (2006). The continuing use of complementary and alternative medicine in South Austra- lia: costs and beliefs in 2004. Medical Journal of Australia, 184, 27-32.

National Centre for Complementary and Alternative Medicine (2006). What is complementary and alternative medicine (CAM)? URL (last checked 15 October 2008).

http://nccam.nih.gov/health/whatiscam/index.htm

O’Connor, E. L., \& White, K. M. (2009). Intentions and willingness to use complementary and alternative medicines: What potential patients believe about CAMs. Complementary Therapy in Clinical Practice, 15, 136-140. doi:10.1016/j.ctcp.2009.03.003

Page, A. C., Jones, R., \& Wilson, F. (2004). Survey of West Australian anxiety support group participants views on treatment processes and outcomes. Australian Psychologist, 39, 208-211. doi:10.1080/00050060412331295090

Pawlak, R., Brown, D., Meyer, M., Connell, C., Yadrick, K., Johnson, J. et al. (2008). Theory of planned behavior and multivitamin supplement use in caucasian college females. The Journal of Primary Prevention, 29, 57-71. doi:10.1007/s10935-008-0127-y

Satia-Abouta, J., Kristal, A. R., Patterson, R. E., Littman, A. J., Stratton, K. L., \& White, E. (2003). Dietary supplement use and medical conditions: The VITAL study. American Journal of Preventive Medicine, 24, 43-51. doi:10.1016/S0749-3797(02)00571-8

Wilson, L. M., \& White, K. M. (2011). Integrating complementary and alternative therapies into psychological practice: A qualitative analysis. Australian Journal of Psychology, 63, 232-242. doi:10.1111/j.1742-9536.2011.00022.x

Wilson, L. M., \& White, K. M. (2008). Identifying the attitudinal, normative, and control beliefs underlying psychologists' willingness to integrate complementary and alternative therapies into psychological practice. In J. Summers (Ed.), Psychology leading change 2008: Proceedings of the 43rd APS annual conference, Hobart.

Yardley, L., Fahmy, S., Jamie, A., \& Furnham, A. (1999). Health beliefs and preferences for medical treatment: A comparison between medical and social science students. Complementary Therapies in Medicine, 7, 101-109. doi:10.1016/S0965-2299(99)80088-0 